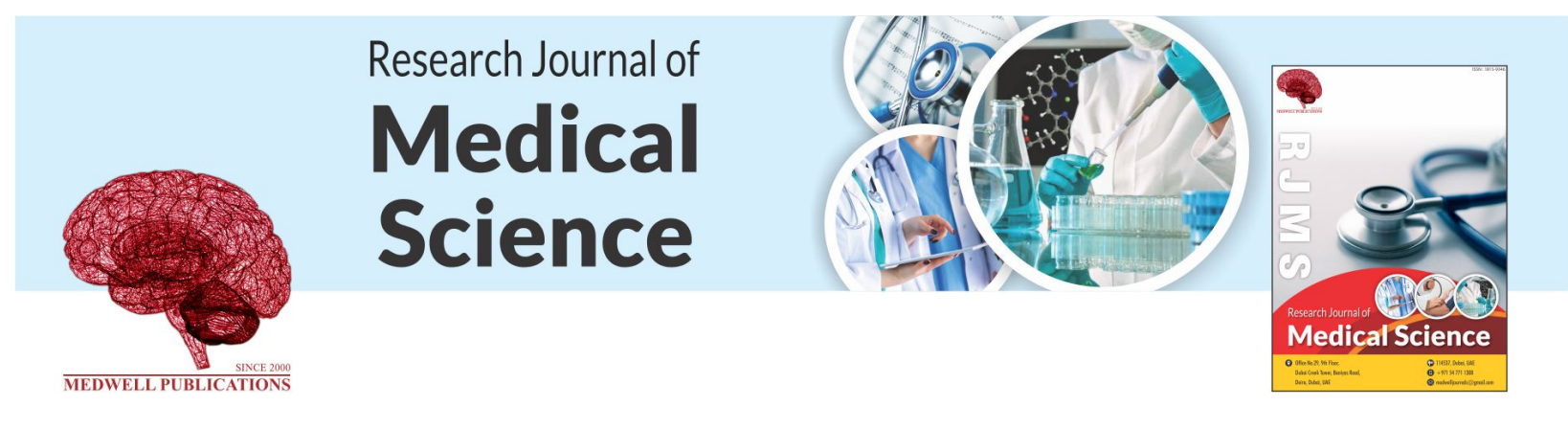

\title{
Do Healthcare Professional View Integrated Management of Childhood Illness Effective? Findings from in-Depth Interviews and Focus Group Discussion in Oman
}

Fannah Abdullah Alfannah

Ministry of Health, Integrated Management Childhood Illness Expert, Department of Public Health, Muscat, Oman

Key words: Integrated Management of Childhood Illness (IMCI), Childhood illness, Primary Health Care (PHC), In-Depth Interviews (IDI), Focus Group Discussion (FGD), Knowledge Attitudes and Skills (KAS)

\section{Corresponding Author:}

Fannah Abdullah Alfannah

Ministry of Health, Integrated Management Childhood Illness Expert, Department of Public Health, Muscat, Oman

Page No.: 1-8

Volume: 14, Issue 01, 2020

ISSN: 1815-9346

Research Journal of Medical Sciences

Copy Right: Medwell Publications
Abstract: This study highlights the findings of a recent research effort where in-depth interviews and focus group discussion were employed to examine views of healthcare professionals on the likely impact of Integrated Management of Childhood Illness (IMCI) on healthcare leadership in Blinded region, Oman. In-Depth Interviews (IDIs) and Focus Group Discussion (FGD) were chosen as appropriate methodologies to collect and analyze data. These methods suited the aim of the study and $12(\mathrm{n}=12)$ healthcare professionals ( 8 male and 4 female) were recruited, having more than 10 years of experience in integrated management of childhood illness. In accord with the best practice of qualitative research methodology, a specific question was developed to initiate discussion and gather participant's knowledge and perceptions. The focal question was whether IMCI strategy could have a positive impact on healthcare leadership. The study was carried out at Blinded Hospital, Sultanate of Oman in April, 2017. All participants approved of IMCI strategy and believed that there is a positive correlation between the strategy and improved child healthcare. In addition, all participants shared positive views on the impact of IMCI on leadership in the healthcare sector. Participants of the study strongly believed that IMCI strategy has: lead to enhanced strategic leadership and management at a macro level; improved Knowledge, Attitudes and Skills (KAS) of Primary Healthcare (PHC) workers, resulted in better prescription practices, prioritized the needs of Primary Healthcare (PHC) units, improved clinical practices and reduced unnecessary referrals. Results from the qualitative data analysis highlight the effectiveness of IMCI strategy. The findings also indicate that IMCI strategy has a positive impact on critical areas such as case management, KAS of healthcare professionals and child healthcare leadership. 


\section{INTRODUCTION}

Childhood illnesses continue to be a challenge in the area of pediatric healthcare, consequently adding to the global disease burden ${ }^{[1]}$. The Integrated Management of Childhood Illness (IMCI) strategy was introduced by the World Health Organization (WHO) in the year $1996^{[2]}$. The strategy has been promoted, globally with an aim to improve child healthcare ${ }^{[4]}$. It focuses on improving healthcare professional's Knowledge, Attitudes and Skills (KAS) as well as refining healthcare systems by following IMCI-recommended practices ${ }^{[4,5]}$. A high majority of sick children (approximately 60\%) can be saved using affordable and evidence-based medical interventions ${ }^{[4,5]}$. We propose that IMCI strategy provides an opportunity to improve child healthcare leading to higher survival rate in low and middle-income countries ${ }^{[6]}$. IMCI strategy is designed in a way that it guides healthcare professionals in effectively assessing and managing childhood illnesses by paying attention to the actual cause of the illness ${ }^{[7]}$. In other words, healthcare professionals who are trained with IMCI focus on identifying and classifying an illness based on its severity by using the IMCI algorithm. This approach helps healthcare professionals to adhere with case-specific treatment. The IMCI algorithm was developed in a rather simple way through employing a series of questions that can help diagnose critical symptoms $^{[8,9]}$.

IMCI can be seen as a framework that is based on demonstrated public health interventions ${ }^{[9]}$. The strategy has been implemented in more than 100 countries followed by several Multi-Country Evaluations (MCEs). Outcomes of the MCEs indicate its effectiveness in improving child healthcare standards for example, improved healthcare professional performance ${ }^{[10]}$. Literature indicates that IMCI-trained healthcare professionals tend to provide accurate and timely treatment and guidance to sick children and their caregivers, respectively ${ }^{[9]}$. Notwithstanding such findings it is observed that anticipated results are yet to be achieved. This is because childhood mortality rates remain high despite global adoption and implementation of IMCI strategy ${ }^{[1]}$.

Findings from more recent studies reveal that some healthcare professionals do not comply with the IMCI-recommended practices ${ }^{[12]}$. It could be deduced that healthcare professionals with more traditional views have a tendency to rely on personal skills and practices while making case assessments and diagnoses. If this assumption stands true there is a manifest need to examine and publish knowledge regarding healthcare professional's views on potential impact of IMCI on child healthcare, strategic leadership and management of healthcare systems.
We argue that child survival is subject to how healthcare professionals perceive and practice IMCI methodology. Indeed, the knowledge and skills that they receive while being trained are important; however, perceptions can significantly influence actual practice of the acquired skills. Improved child healthcare requires a paradigm shift at the strategic level ${ }^{[13]}$ and it is necessary that new interventions are fully understood, implemented and practiced. It might be, so that, IMCI-trained healthcare professionals do not fully comply with the guidelines because they face a dynamic environment on a daily basis or might receive lack of supervision. It is emphasized over and again that appropriate supervision can help improve adherence leading to enhanced performance by bridging the gap between knowledge and clinical practice ${ }^{[14]}$. Healthcare professionals and policy makers who perceive IMCI as being an effective strategy can become opinion leaders and further promote its practice in a wider context.

Significant number of research articles highlights positive impact of IMCI on improved child healthcare. In Tanzania a $13 \%$ reduction in childhood mortality has been linked with IMCI-recommended practices ${ }^{[15]}$. Practice of IMCI-recommended guidelines in clinical settings is also accepted as a deciding factor for improving child healthcare standards in other countries ${ }^{[16,20]}$. Further, literature indicates a positive correlation between IMCI-guidelines and improved community health practices, correct prescriptions, reduced unnecessary referrals and better case assessment skills ${ }^{[17,20,21]}$. Despite evident benefits of IMCI-recommended guidelines and practices, optimal results are yet to be achieved.

One step towards this direction would be to explore and understand how healthcare professionals foresee the scope of IMCI-training at a strategic level. We assume that when healthcare professionals perceive IMCI as an effective intervention they will support the strategy and encourage its implementation. In this study, we describe the perceptions and experiences of $12(n=12)$ healthcare professionals with a specific focus on the likely impact of IMCI strategy on improving strategic leadership and management in child healthcare sector. To the best of our knowledge, no previous study has examined healthcare professional's perceptions and experiences from this point of view.

\section{MATERIALS AND METHODS}

The researcher conducted In-Depth Interviews (IDIs) and a Focus Group Discussion (FGD) with IMCI-trained healthcare professionals to explore their views and perceptions about IMCI strategy and whether they believed that the strategy has made a positive impact on child healthcare in the region. The IDIs and FGD were 
conducted at Blinded Hospital, Oman in April, 2017. Ethical approval was obtained from the Ministry of Health, Oman prior to the study. All participants of the study had $>10$ years of experience in IMCI and were working different capacities in blinded region. Blinded region was selected for the study for being a leader in IMCI implementation in the Sultanate. The IMCI focal point for blinded region was contacted prior to the study. The regional focal point helped in conducting the interviews as well as focus group discussion. We carefully selected the participants based on their experience with IMCI training and practice. This was done to ensure that their replies would provide rich insights and broaden the discussions. This particular approach is in line with purposive sampling strategies ${ }^{[22]}$. This particular strategy helps researchers understand important cases rather than general inferences from a set of specific population samples $^{[23]}$.

IDIs were chosen for collecting qualitative data because the technique helps conducting detailed interviews with a relatively small number of participants in order to understand their opinions on a particular subject or research question ${ }^{[24,25]}$. Further, this technique helps gather data about participant's perceptions, experiences and expectations about a specific intervention. In-depth interviews are also known for being highly useful when researchers aim to get detailed insights and understand individual opinions. At times it is also argued that this technique is suitable for situations where conducting a focus group is not practical ${ }^{[24]}$.

In addition to the in-depth interviews we opted to conduct a FGD. This research methodology was chosen as we had a small number of participants and our objective was to discuss a specific topic to gather and generate further ideas. Unlike individual in-depth interviews, focus groups can provide an ideal platform for interaction between different participants. Focus group discussions are generally, accepted as a data collection technique in the area of health and medical research ${ }^{[26]}$. It is often argued that focus groups discussions lead to detailed responses when compared with direct questions asked in individual interviews ${ }^{[27]}$. As qualitative research is known for being a heterogeneous area where researchers are often left with no choice but to make certain assumptions ${ }^{[28]}$ focus groups are known for being an effective research technique as they can be seen as a group interview where carefully selected participants share their opinions, experiences, perceptions and attitudes towards a specific topic or area of research ${ }^{[28]}$. The number of participants in the presented study was 12 $(\mathrm{n}=12)$ which is relatively small but large enough for each participant to make significant contribution to the discussion as proposed by Krueger and Casey ${ }^{[29]}$.
Table 1: Basic demography of the participants from Blinded region

\begin{tabular}{ll}
\hline Variables & Participants (N) \\
\hline Gender & 8 \\
Male & 4 \\
Female & \\
Age & $40-49$ \\
Years & \\
Occupation/Position & 1 \\
Health services (regional director) & 1 \\
Primary health care (regional director) & 3 \\
IMCI expert & 2 \\
Pediatrician & 1 \\
General physician & 2 \\
Health education (regional in-charge) & 1 \\
Nursing (regional in-charge) & 1 \\
Pharmacist (regional in-charge) & $>15$ years \\
Experience in IMCI strategy &
\end{tabular}

The overall research approach was qualitative and descriptive in nature. Healthcare professionals from blinded region were selected for participation and invited to partake the study. All the invites agreed to participate in the study on a voluntary basis. The objective of the study was explained to the participants and informed consent was obtained following international best practice. Prior to the actual study, we ran a pilot for both IDIs and FDG to ensure that the questions and responses would produce pertinent interactive discussions. Running a pre-study pilot helped us refine key research questions in addition to providing hands on training to the focus group moderator and note taker who plays a central role $^{[28,29]}$ in such a research approach. The structure and content of both IDIs and FGD were prepared with the help of a healthcare researcher. A relatively simple form was also prepared with four items, i.e. age, gender, occupation and experience in IMCI. For data analysis, we wrote down transcriptions and mapped common topics that were later used as representative quotes. All the recorded information and data were dealt with strict confidentiality and used for the purpose of the study only. Table 1 exhibits some basic details about the participants.

\section{RESULTS AND DISCUSSION}

In-Depth Interviews (IDIs): Nearly all the participants gave positive remarks about the impact of IMCI strategy and its benefits. They spoke about improved treatment standards, improvement in accurate and timely diagnosis, lower numbers of unnecessary referrals, better treatments at PHC level and so on. In addition, IDIs revealed that there is a positive correlation between IMCI-recommended practices and improved strategic leadership and management of healthcare system. In general, findings from the interviews indicate a positive correlation between IMCI and improved child healthcare in the region. Below are some representative quotes: 
"There is a significant and noticeable reduction in unnecessary prescriptions which $\mathrm{i}$ feel is a direct consequence of complying with IMCI guidelines"

"IMCI guidelines are easy enough for today's medical professionals. It is my personal observation that IMCI-trained doctors have a higher level of knowledge and understanding. Therefore, they focus on finding the root cause rather than coming up with assumptive diagnosis and writing prescriptions"

In response to the impact of reduced prescriptions and consumptions of drugs, the participants approved of the role of IMCI.

"Now that, we have IMCI-trained healthcare professionals, the positive implications can be seen at the pharmaceutical level. Even the pharmacies are receiving more attention in terms of improving infrastructure, storage capacities etc."

"The fruits of implementing IMCI can be seen at both macro and micro levels in the region. If you visit any of the PHC facilities, you will find a well-organized human resource allocation, improved infrastructure, lower turn over rates and more satisfied caregivers. I find this exciting as our aim is to provide sick children with immediate but effective medical care and surely IMCI guidelines facilitate the process"

Responding to the question about KAS, all participants gave strongly positive remarks.

"It is a result of the training and experience of applying IMCI guidelines that one can see an overall improvement in knowledge, attitudes and skills of pediatricians when they are diagnosing a sick child and at the same time counseling his/her parents"

"There is no doubt in my mind that IMCI strategy has improved the KAS of both medics (doctors) and paramedic (nursing staff). Healthcare units have (consequently) become effective, efficient and easier to manage. We are in a better position to provide immediate and accurate medical treatment to sick children. I guess, I would not be wrong if I were to say that IMCI guidelines and their actual implementation can help us develop an integrated healthcare system in the near future where we will have better tools, techniques and appropriate channels of communication for furthering the healthcare facilities and services"

Focus Group Discussion (FGD): Focus group discussion was conducted to get a deeper understanding of how the participants responded to the questions during their interaction with other specialists/experts from the same region. Focus group discussions are frequently used to understand people's viewpoints and to obtain clarifications that are relatively hard to get by asking direct questions ${ }^{[27]}$.

Focus group discussions suit healthcare research in particular as majority of health-related conditions are a consequence of societal situations ${ }^{[30]}$. Lastly, advantages that are generally associated with focus group discussions include but are not limited to exchange of expert opinions, easily recordable discussions and collection of data in the form of ideas, suggestions and viewpoints about a specific issue. Following are the identified themes that emerged during the focus group discussion:

Improved knowledge, attitudes and skills: All the participants responded with positive remarks in response to the question, "Do you think that IMCI strategy has any impact on the knowledge, attitudes and skills of healthcare professionals?" Below are some of the representative quotes:

"There is an evident improvement in the knowledge and skills of healthcare workers as a result of practicing IMCI-guidelines. My observation is based on several years and now that I compare practitioners who have experience in IMCI, I find them with enhanced skills especially in case assessment". (Director General for Healthcare, Blinded Region)

"As a senior manager in the healthcare sector, I have observed that healthcare professionals, both doctors and nurses, have an improved approach when treating a (sick) child. This, in my opinion, can be attributed to an integrated approach of teaching and applying child healthcare strategy (IMCI)." (Director of PHC, Blinded Region)

"I feel confident when I am dealing with a sick child. I have no doubt that over the years, IMCI guidelines have helped me improve my professional skills especially in making accurate diagnosis". (IMCI-trained Pediatrician, Blinded Region) 
Reduced drug consumption: An overwhelming number of positive remarks were received in response to the question, "Is there an impact of IMCI strategy on drug consumption?" Some of the prominent responses are listed below:

"Definitely! The amount of drug consumption is dropped down significantly over the past years. This is indeed a positive outcome of practicing IMCI strategy and guidelines" (In-charge of Pharmaceutical Department, Blinded Region)

"It (IMCI) has helped me follow a systematic path (algorithm) to diagnose the root cause of an illness. Once a systematic diagnosis is made, it helps prescribing drugs specific to the illness. So, I would be surprised if the overall drug intake is not reduced." (IMCI-trained Pediatrician, Blinded Region)

"Yes, there is a noticeable reduction in drug consumption in our region. This outcome has multiple benefits. For me, I would say that reduced drug intake (consumption) means that I have more resources available for improving the hospital (healthcare) infrastructure, especially, the availability of essential drugs at pharmacies". (Director of Primary Health Care, Blinded Region)

"Yes, there is a noticeable reduction in drug consumption in our region. This outcome has multiple benefits. For me, I would say that reduced drug intake (consumption) means that I have more resources available for improving the hospital (healthcare) infrastructure, especially, the availability of essential drugs at pharmacies". (Director of Primary Health Care, Blinded Region)

Allocation of resources: Improved resource allocation was commonly voiced in response to the question "Is there a relation between IMCI strategy and resource management?" Some of the representative quotes are stated below:

"The strategy (IMCI) has a wider impact on the overall healthcare system. The benefits are not just limited to a specific part (unit) of the healthcare structure. As a matter of fact, the benefits are far reaching. As a result of intake (consumption), I find myself in a better position to allocate resources to areas that need them most. For example, I can allocate budget for infrastructure, timely and adequate supplies of drugs and training needs of medical staffs". (Director General Healthcare General for Healthcare, Blinded Region)

"I have access to more resources now as compared with what I had about 10 years back. This is not just a coincidence. It is because we are following an integrated (IMCI) approach". (Director General for Primary Health Care, Blinded Region)

"It has become easier to reach out to the senior managers when it comes to planning our day to day operations. I believe the (IMCI) guidelines have made our daily operations simpler and effective." (In-charge of Pharmaceutical Department, Blinded Region)

General comments: When given an opportunity to discuss the impact of IMCI strategy on the regional healthcare system, all the participants were vocal and shared their views. The views revealed that the participants largely approved of the strategy. They highlighted the positive impact of the strategy on a macro level. Some of the prominent quotes are presented below:

"The impact of IMCI teaching and practicing on improving referrals is manifest. From the time that, we started following (practicing) the strategy in the region, there is a noticeable reduction in unnecessary referrals". (IMCI-expert, Blinded Region)

"The benefits are for everyone. Paramedic staffs that are trained with IMCI have better skills as compared with those who were trained with the vertical teaching style. However, the management has encouraged and supported all to get (IMCI) training. I also want to add that now (today) both medical doctors and nurses have become better counselors. They give better advice to the caregivers and as a result we witness higher number of follow up visits". (In-charge of Nursing Education and Training, Blinded Region)

"IMCI trained healthcare professionals are more confident. It is my understanding that they feel empowered and skilled for performing their duties effectively. This makes life easier for a 
senior manager, for example, a healthcare unit in-charge who has a skilled team can spend more time on planning and implementing strategic decisions." (Director General of Primary Health Care, Blinded Region)

"When we talk about sick children, we simply cannot overlook their parents (immediate caregivers). IMCI guidelines have made our doctors and nurses better at communication and giving advice. This in turn has resulted in building a stronger relationship between the community and healthcare professionals. They (parents/immediate caregivers) not only listen to the advice but also act on it. The action can be traced when the make follow up visits. I am convinced that community has more confidence in healthcare facilities that we now provide." (Director General for Healthcare, Blinded Region)

"I have no doubt that IMCI implementation has resulted in better management of resources, cost effectiveness and improved health indicators". (Director of Primary Health Care, Blinded Region)

\section{RESULTS AND DISCUSSION}

This study was performed to explore views, perceptions and experiences of healthcare professionals about the impact of IMCI-strategy on healthcare system as well as strategic leadership. The participants included senior healthcare managers, medical practitioners, IMCI experts, pediatricians and nursing educators. All participants approved of IMCI-strategy and acknowledged its positive impact on the overall healthcare standard. This finding is similar to other research findings that have identified benefits of implementing IMCI-strategy ${ }^{[31,33]}$ where improved KAS, better diagnostic practices and enhanced community engagement are often discoursed. All participants agreed that IMCI being a holistic approach for managing child healthcare has multiple benefits. Participants also acknowledged that the approach is far more effective when compared with the traditional (vertical) teaching methodology when it comes to diagnosing a sick child and making accurate diagnosis.

According to the participants, IMCI strategy has significantly reduced unnecessary referrals and drug consumption and as a consequence there are more resources available for health care managers to focus on making strategic decisions related with improving infrastructure, planning human resources and engaging community, especially, immediate caregivers. These findings are similar to MCEs that have highlighted similar outcomes $^{[34,35]}$. From the in-depth interviews and focus group discussion it is observed that healthcare professionals perceive IMCI strategy as an effective initiative that not only improves child healthcare but has direct implications for healthcare managers.

For example, it was repeatedly reported that healthcare managers are in a better position for allocating resources at primary health care units. Further, it was highlighted that even the pharmacies are well equipped now as a consequence of IMCI strategy implementation. Almost all the participants supported IMCI as a key contributor for improving strategic leadership in the healthcare sector. They frequently related accurate diagnosis with reduced drug consumption which in their opinion makes more resources available to healthcare managers for improving related areas such as improving the healthcare facilities, providing IMCI training to new staff members and engaging with the community more effectively. Indeed, community engagement remains an essential part of the IMCI strategy ${ }^{[36]}$.

\section{CONCLUSION}

The study highlights that Integrated Management of Childhood Illness (IMCI) strategy is acknowledged as an effective technique for improving child healthcare. Likewise, the findings indicate that there is a positive correlation between IMCI-teaching strategy and improved strategic child healthcare leadership. Lastly, the findings indicate that when implemented and practiced, although, it might take sometime, IMCI benefits the entire healthcare system including primary healthcare units, immediate caregivers and indeed sick children.

\section{ACKNOWLEDGEMENTS}

The researcher would like to thank all of the participants for their cooperation as well as the relevant authorities of the Ministry of Health, Oman. In addition, the researcher appreciates the efforts of the healthcare professionals who aided in the development and validation of the questionnaire items.

\section{LIMITATIONS}

This study has its limitations. First, it was performed in one region that is known to be among the leaders in practicing IMCI-recommended guidelines. Therefore, at present it is not possible to generalize the findings. Secondly, we did not include a community representative in the study, therefore, a potential bias cannot be overruled. Lastly, the sample size is evidently small; however, in-depth interviews and especially focus group discussions support such a sample size, especially when the participants are specialists/experts in a particular area. 
We do however, emphasize that the prime objective of the study was to examine the views of healthcare professionals about the effectiveness of IMCI strategy. It is for this reason that the results highlight rather descriptive responses.

\section{IMPLICATIONS}

While the implications for practice might be observed as beyond the findings of the study, they do nevertheless pave the way for further studies at a larger scale. Therefore we plan to perform another study where majority of the participants will be from the community. It would be interesting to collect their responses and compare with the findings of this study.

\section{REFERENCES}

01. Al-Araimi, F.A. and S.U. Langrial, 2016. A hypothetical model to predict nursing student's perceptions of the usefulness of pre-service integrated management of childhood illness training. Sultan Qaboos Univ. Med. J., 16: 469-474.

02. Goga, A.E., L.M. Muhe, K. Forsyth, M. Chopra and S. Aboubaker et al., 2009. Results of a multi-country exploratory survey of approaches and methods for IMCI case management training. Health Res. Policy Syst., 7: 1-18.

03. Tulloch, J., 1999. Integrated approach to child health in developing countries. Lancet, 354: SII16-SII20.

04. Bryce, J., R .E. Black, N. Walker, Z.A. Bhutta and J.E. Lawn et al., 2005b. Can the world afford to save the lives of 6 million children each year?. Lancet, 365: 2193-2200.

05. Bryce, J., C. Boschi-Pinto, K. Shibuya and R.E. Black, 2005a. WHO estimates of the causes of death in children. Lancet, 365: 1147-1152.

06. Victora, C.G., K. Hanson, J. Bryce and J.P. Vaughan, 2004. Achieving universal coverage with health interventions. Lancet, 364: 1541-1548.

07. Gove, S., 1997. Integrated management of childhood illness by outpatient health workers: Technical basis and overview, The WHO Working Group on Guidelines for Integrated Management of the Sick Child. Bull. World Health Organ., 75: 7-24.

08. Zucker, J.R., B.A. Perkins, H. Jafari, J. Otieno and C. Obonyo et al., 1997. Clinical signs for the recognition of children with moderate or severe anaemia in western Kenya. Bull. World Health Organ., 75: 97-102.

09. Horwood, C., A. Voce, K. Vermaak, N. Rollins and S. Qazi, 2009. Experiences of training and implementation of Integrated Management of Childhood Illness (IMCI) in South Africa: A qualitative evaluation of the IMCI case management training course. BMC. Pediatr., 9: 1-9.
10. De-Savigny, D., A.S. Joanna, J. Bryce, T. Lambrechts and C. Mbuya, 2004. Effect of integrated management of childhood illness on observed quality of care of under-fives in rural Tanzania. Health Policy Plann., 19: 1-10.

11. Lange, S., A. Mwisongo and O. Maestad, 2014. Why don't clinicians adhere more consistently to guidelines for the Integrated Management of Childhood Illness (IMCI)?. Soc. Sci. Med., 104: 56-63.

12. Ahmed, H.M., M. Mitchell and B. Hedt, 2010. National implementation of Integrated Management of Childhood Illness (IMCI): Policy constraints and strategies. Health Policy, 96: 128-133.

13. Locock, L., S. Dopson, D. Chambers and J. Gabbay, 2001. Understanding the role of opinion leaders in improving clinical effectiveness. Soc. Sci. Med., 53: 745-757.

14. Chaudhary, N., P.N. Mohanty and M. Sharma, 2005. Integrated management of childhood illness (IMCI) follow-up of basic health workers. Indian J. Pediatr., 72: 735-739.

15. Bryce, J., C.G. Victora, J.P. Habicht, J.P. Vaughan and R.E. Black, 2004. The multi-country evaluation of the integrated management of childhood illness strategy: Lessons for the evaluation of public health interventions. Am. J. Public Health, 94: 406-415.

16. Amaral, J., A.J. Leite, A.J. Cunha and C.G. Victora, 2005. Impact of IMCI health worker training on routinely collected child health indicators in Northeast Brazil. Health Policy. Plann., 20: i42-i48.

17. Pariyo, G.W., E. Gouws, J. Bryce and G. Burnham, 2005. Improving facility-based care for sick children in Uganda: Training is not enough. Health Policy Plann., 20: i58-i68.

18. Arifeen, S.E., D.E. Hoque, T. Akter, M. Rahman and M.E. Hoque et al., 2009. Effect of the Integrated Management of Childhood Illness strategy on childhood mortality and nutrition in a rural area in Bangladesh: A cluster randomised trial. Lancet, 374: 393-403.

19. Wagstaff, A., F. Bustreo, J. Bryce and M. Claeson, 2004. Child health: Reaching the poor. Am. J. Publ. Health, 94: 726-736.

20. Naimoli, J.F., A.K. Rowe, A. Lyaghfouri, R. Larbi and L.A. Lamrani, 2006. Effect of the Integrated Management of Childhood Illness strategy on health care quality in Morocco. Intl. J. Q. Health Care, 18: 134-144.

21. Ebuehi, O.M., 2009. Health care for under-fives in Ile-Ife, South-West Nigeria: Effect of the Integrated Management of Childhood Illnesses (IMCI) strategy on growth and development of under-fives. Afr. J. Primary Health Care Family Med., 1: 62-70. 
22. Short, J.C., D.J. Jr. Ketchen and T.B. Palmer, 2002. The role of sampling in strategic management research on performance: A two-study analysis. J. Manage., 28: 363-385.

23. Patton, M.Q., 1999. Enhancing the quality and credibility of qualitative analysis. Health Ser. Res., 34: 1189-1208.

24. Boyce, C. and P. Neale, 2010. Conducting in-depth interviews: A guide for designing and conducting in-depth interviews for evaluation input. Monit. Eval., 2: 1-16.

25. Turner, D.W., 2010. Qualitative interview design: A practical guide for novice investigators. Qual. Rep., 15: 754-760.

26. Wong, L.P., 2018. Focus group discussion: A tool for health and medical research. Singapore Med. J., 49: 256-260.

27. Kitzinger, J., 1995. Qualitative research: Introducing focus groups. Br. Med. J., 311: 299-302.

28. Freeman, T., 2006. Best practice in focus group research: Making sense of different views. J. Adv. Nurs., 56: 491-497.

29. Krueger, R.A. and M.A. Casey, 2000. Focus Groups: A Practical Guide for Applied Research. 3rd Edn., Sage Publishing, Thousand Oaks, California, Pages: 193.

30. Carter, S. and L. Henderson, 2005. Approaches to Qualitative Data Collection in Social Science. In: Handbook of Health Research Methods: Investigation, Measurement and Analysis, Ebrahim, S. and A. Bowling (Eds.). McGraw-Hill Education, New York, USA., ISBN: 97803352243641, pp: 215-230.
31. Weber, M.W., E.K. Mulholland, S. Jaffar, H. Troedsson and S. Gove et al., 1997. Evaluation of an algorithm for the Integrated Management of Childhood Illness in an area with seasonal Malaria in the Gambia. Bull. World Health Organ., 75: 25-32.

32. Acuin, J., 2004. Chronic Suppurative Otitis Media: Burden of Illness and Management Options. World Health Organization, Geneva, Switzerland, ISBN:9241591587, Pages: 83.

33. Harkins, T., C. Drasbek, J. Arroyo and $M$. McQuestion, 2008. The health benefits of social mobilization: Experiences with community-based Integrated Management of Childhood Illness in Chao, Peru and San Luis, Honduras. Promotion Educ., 15: 15-20.

34. Mushi, H.P., K. Mullei, J. Macha, F. Wafula and J. Borghi et al., 2010. The challenges of achieving high training coverage for IMCI: Case studies from Kenya and Tanzania. Health Policy Plann., 26: 395-404.

35. Gouws, E., J. Bryce, J.P. Habicht, J. Amaral and G. Pariyo et al., 2004. Improving antimicrobial use among health workers in first-level facilities: Results from the multi-country evaluation of the Integrated Management of Childhood Illness strategy. Bull. World Health Organ., 82: 509-515.

36. Abdel-Rahman, S.H. and S.M. Alfadil, 2008. Introducing the IMCI community component into the curriculum of the Faculty of Medicine, University of Gezira. Eastern Mediterranean Health J., 14: 731-741. 